\title{
Model Reduction of Multidimensional and Uncertain Systems
}

\author{
Carolyn L. Beck, John Doyle, and Keith Glover, Fellow, IEEE
}

\begin{abstract}
Model reduction methods are presented for systems represented by a linear fractional transformation (LFT) on a repeated scalar uncertainty structure. These methods involve a complete generalization of balanced realizations, balanced Gramians, and balanced truncation model reduction with guaranteed error bounds, based on solutions to a pair of linear matrix inequalities (LMI's) which generalize Lyapunov equations. The resulting reduction methods immediately apply to uncertainty simplification and state order reduction in the case of uncertain systems but also may be interpreted as state order reduction for multidimensional systems.
\end{abstract}

\section{INTRODUCTION}

T THE process of modeling systems and designing controllers using modern robust control methods often results in models that have high state order and complicated uncertainty descriptions. These models may be expensive to implement and difficult to analyze, in particular when further interconnected in more complex systems. However, there have been no systematic methods proposed for reducing the uncertainty descriptions. In contrast, for models without uncertainty, there exist numerous well-known state order reduction methods and associated error bounds, examples of which include the balanced model reduction method and its additive $H_{\infty}$ norm error bound [15], [18], [21], [26]; the optimal Hankel norm model reduction method and its Hankel norm error bound [18]; and the balanced stochastic truncation model reduction method and its relative $H_{\infty}$ norm error bound [13]. The balanced truncation model reduction method was first extended to uncertain systems in [32]. We review this reduction method and the related error bounds, providing simplified proofs. We then give a new model reduction method for uncertain systems that relies on the solution of two linear matrix inequalities (LMI's) and results in tighter error bounds than the balanced truncation method. These new results, which were first noted in [7], are based on technical machinery presented in [27]. Similar results for standard onedimensional (1-D) continuous systems are given in [23] using quite different methods. A related necessary and sufficient

Manuscript received March 17, 1995; revised April 20, 1996. Recommended by Associate Editor, J. Shamma.

C. L. Beck was with the Electrical Engineering Department, California Institute of Technology, Pasadena, CA 91125 USA. She is now with the Department of Electrical Engineering, University of Pittsburgh, Pittsburgh, PA 15213 USA.

J. Doyle is with Control and Dynamical Systems, California Institute of Technology, Pasadena, CA 91125 USA.

$\mathrm{K}$, Glover is with the Department of Engineering, University of Cambridge, Cambridge, CB2 1PZ, UK.

Publisher Item Identifier S 0018-9286(96)07694-5. condition for exact reducibility, the converse of minimality, of uncertain systems may also be stated [3], [5], [6].

In this paper, we are mainly concerned with uncertain systems that are modeled by a linear fractional transformation (LFT) on a repeated scalar uncertainty structure. In the most abstract formulation these uncertainties are simply noncommuting indeterminates, as in formal power series. However, to study model reduction with error bounds, we need additional structure in the form of a norm, and it is convenient to assume that the uncertainty is made up of arbitrary time-varying scalar operators on $l_{2}$ and that the model reduction errors are measured as $l_{2}$-induced norms. Under these assumptions, the bounds in this paper are tight, in a sense that will be made precise. We may also apply the bounds to system models where the uncertainty has further structure, such as timeinvariance or real parametric variance, or when the repeated scalar uncertainty is viewed as multiple transform variables in a multidimensional system. In these latter examples the bounds are, in general, conservative.

Basic background material is presented in Section II, including our notation and a review of LFT representations of uncertain systems. In Section III, we review balanced realizations, minimality, and the error bounds associated with balanced truncation model reduction for the 1-D case. In Section IV, uncertain systems are considered: balanced realizations, stability, and norms are discussed, and error bounds for balanced truncation of uncertain systems are given. In Section V, the new LMI reduction method for uncertain system realizations is presented.

\section{PRELLIMINARIES}

The notation we use is as follows: $l_{2}$ denotes the space of sequences which are square summable, and $\mathcal{L}\left(l_{2}\right)$ represents the space of all linear time-varying (LTV) operators on $l_{2}$. We represent matrices in the real and complex fields by $\mathbb{C}^{n \times m}$ and $\mathbb{R}^{n \times m}$ and the integers by $\mathbb{Z}$. The shift operator on $l_{2}$ is denoted by $\lambda$, and the identity matrix is denoted by $I$. The maximum singular value of $A \in \mathbb{C}^{n \times m}$ is denoted by $\bar{\sigma}(A)$, and the minimum eigenvalue by $\lambda_{\min }(A) ; A^{*}$ denotes the complex conjugate transpose. The dimensions of a matrix $A$ are denoted $\operatorname{dim}(A)$. For notational convenience, dimensions will not be given unless pertinent to the discussion.

The main focus of robust control has been to evaluate the effects of uncertainty when analyzing and designing controllers for linear systems. The motivation for this approach is that system performance is often dominated by the uncertainty in a model; designs based on an ideal mathematical model 


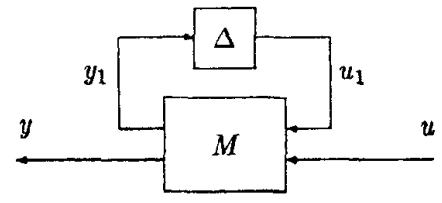

Fig. 1. Uncertain system.

frequently perform poorly on the real system. The source of such uncertainty might be unknown nonlinearities, unmodeled dynamics, disturbances, parameter variations, or any combination of the aforementioned. As a mathematical representation for uncertainty in system models, we use the LFT paradigm which is represented pictorially in Fig. 1 and described below.

Typically, $M$ represents the nominal system model which is often assumed to consist of the transfer function for the plant plus system weighting functions, and $\Delta$ represents the uncertainty. Because each perturbation source is likely to enter the real system at a different location, collecting these into one uncertainty block results in $\Delta$ having a diagonal block structure. Furthermore, the perturbations are often assumed to be norm-bounded operators and frequently have additional structure such as time-invariance or real parametric variance.

In the LFT models we consider, the shift operator $\lambda$ corresponding to the system transform variable is also included in $\Delta$, the uncertainty block; that is, the transfer relations for the plant and weighting are explicitly written as LFT's on the shift operator. Thus, we assume $M$ is a constant matrix and $\Delta$ represents the system uncertainty and shift operators. In particular, we refer to $M$ as the system realization matrix which we partition as

$$
M=\left[\begin{array}{ll}
A & B \\
C & D
\end{array}\right]
$$

and we assume $\Delta$ lies in some prescribed set. In particular, the uncertainty set $\Delta$ we consider in this paper is

$$
\boldsymbol{\Delta}=\left\{\operatorname{diag}\left[\delta_{1} I_{n_{1}}, \cdots, \delta_{p} I_{n_{p}}\right]: \delta_{i} \in \mathcal{L}\left(l_{2}\right)\right\} .
$$

The input/output (I/O) mapping for this system is determined by the LFT

$$
y=(\Delta \star M) u, \quad \Delta \in \Delta
$$

where we assume the system input and output signals, $u$ and $y$, are in $l_{2}$. The Redheffer star product of the system components is

$$
\Delta \star M=D+C \Delta(I-A \Delta)^{-1} B
$$

whenever the inverse is well defined. We will refer to such system models by the pair $(\boldsymbol{\Delta}, M)$. For analysis purposes, we will often consider $\Delta$ which lie in a norm-bounded subset of $\Delta$, that is

$$
\mathbf{B}_{\Delta}=\left\{\Delta \in \Delta:\|\Delta\|_{l_{2} \rightarrow l_{2}} \leq 1\right\}
$$

where $\|\cdot\|_{l_{2} \rightarrow l_{2}}$ denotes the induced norm.

As mentioned previously, the most general way to interpret these system models is to view the $\delta_{i}$ as noncommuting indeterminants; in this case the LFT of (2) is a rational function in several noncommuting variables and thus can also be viewed as a formal power series over the set $\left\{\delta_{1}, \cdots, \delta_{p}\right\}$ by considering the expansion of

$$
\Delta \star M=D+\sum_{k=0}^{\infty} C \Delta(A \Delta)^{k} B .
$$

Since formal power series have been studied in a wide variety of disciplines, this interpretation allows us to formulate connections to established realization theory (see [3] for details), for example to the series minimality conditions and Hankel matrices developed by Fliess [19]. (See [8] for further details and references relating to formal power series.) However, to quantitatively evaluate model reduction methods, we need a less abstract setting. In particular, we consider one of the $\delta_{i}$ as the shift operator in an uncertain discrete-time system. The remaining $\delta_{i}$ are then viewed as LTV operators on $l_{2}$. In this case, we present a condition which is both necessary and sufficient for satisfaction of the tighter model reduction error bounds which are measured in an $l_{2}$ induced norm. A related necessary and sufficient condition under which reduced-order models can be found such that the error between the full and reduced-order models is zero can also be shown. If, instead, the remaining $\delta_{i}$ are time-invariant or real parametric norm bounded perturbations, these conditions are sufficient, but not necessary, and may be somewhat conservative.

Alternatively, we may view the $\delta_{i}$ as different transform variables in a multidimensional system, and thus the $\delta_{i}$ considered are commuting variables, as in the time-invariant case. (See [9] and the references therein for information on multidimensional systems.) In the multidimensional system case, when we reduce the model we reduce the state orders of the model, and thus a system model that may be reduced with no error is said to be reducible, or nonminimal, as in the 1-D state-space case. Generally, we would like to find reducedorder models which match the original model well at all values of the $\delta_{i}$ on the polydisc $\left|\delta_{i}\right|=1$, for all $i$. The results in this paper are directly relevant to this interpretation, as they provide computable sufficient conditions under which reducedorder multidimensional models may be found for which there is little or no error. Again, these methods may be conservative for multidimensional models in that lower order models may exist which are equivalent, or close, to the original model but which cannot be found via the methods presented herein.

Remark 1: Note that throughout this paper we refer to models with $\Delta=\lambda I$ as 1-D systems and henceforth to models with $\Delta$ defined as in (1) simply as uncertain systems.

\section{1-D SYSTEMS}

To facilitate the discussion of model reduction methods for uncertain systems, we begin with a review of Lyapunov equations, balanced realizations, minimality, and balanced truncation model reduction for 1-D system realizations. We state the well-known upper bound for balanced truncation model reduction error originally proven by Enns [15] (see also Glover [18]) for continuous-time systems. We provide a proof of this bound for discrete-time systems which generalizes immediately to uncertain system representations. The proof 
was first presented in [32] and is given in the Appendix in a corrected and more concise form. A more general proof which also encompasses model reduction of continuous-time uncertain systems has recently been developed using integral quadratic constraints (IQC) methods [1] and [30].

We consider finite dimensional, linear time-invariant (LTI) systems of the form

$$
\begin{array}{r}
x(k+1)=A x(k)+B u(k) \\
y(k)=C x(k)+D u(k)
\end{array}
$$

thus $M=\left[\begin{array}{ll}A & B \\ C & D\end{array}\right]$ is the system realization and $\Delta=\lambda I$. For the 1-D case, we denote the system transfer operator by $G:=\lambda I \star M=D+C \lambda(I-A \lambda)^{-1} B$.

\section{A. Balanced Realizations and Reducibility}

Suppose $M$ is a 1-D stable system realization and $Y$ and $X$ are the controllability and observability Gramians, respectively. That is, $Y=Y^{*} \geq 0$ and $X=X^{*} \geq 0$ satisfy the Lyapunov equations

$$
\begin{aligned}
A Y A^{*}-Y+B B^{*} & =0 \\
A^{*} X A-X+C^{*} C & =0 .
\end{aligned}
$$

From standard Lyapunov theory, we know that the pair $(A, B)$ is controllable if and only if $Y>0$, and $(C, A)$ is observable if and only if $X>0$, in which case we say the realization is minimal or irreducible.

Suppose the system realization, $M$, is transformed by the nonsingular matrix $T$ which gives the eigenvector decomposition

$$
Y X=T^{-1} \Lambda T, \quad \Lambda=\operatorname{diag}\left(\lambda_{1}, \cdots, \lambda_{n}\right) .
$$

Since $Y \geq 0$ and $X \geq 0$, it can be shown that $Y X$ has a real diagonal Jordan form and that $\Lambda \geq 0$. If $M$ is a minimal realization, $T$ can always be chosen such that

$$
\begin{aligned}
\hat{Y} & =T Y T^{*} \\
& =\Sigma
\end{aligned}
$$

and

$$
\begin{aligned}
\hat{X} & =\left(T^{-1}\right)^{*} X T^{-1} \\
& =\Sigma
\end{aligned}
$$

where $\Sigma=\operatorname{diag}\left(\sigma_{1}, \sigma_{2}, \cdots, \sigma_{n}\right)>0$ and $\Sigma^{2}=\Lambda$. The transformed realization

$$
\hat{M}=\left[\begin{array}{cc}
T A T^{-1} & T B \\
C T^{-1} & D
\end{array}\right]
$$

with controllability and observability Gramians $\hat{Y}=\hat{X}=\Sigma$, is referred to as a balanced realization. Recall that $G=$ $\lambda I \star M=\lambda I \star \hat{M}$.

More generally, if the realization of a 1-D system is not minimal, then there exists a transformation such that the controllability and observability Gramians are diagonal, and the controllable and observable subsystem is balanced. The following theorem is standard, so the proof is omitted.
Theorem 2: For any stable system realization $M=\left[\begin{array}{cc}A & B \\ C & D\end{array}\right]$ there exists $T$ such that

$$
\hat{M}=\left[\begin{array}{cc}
T A T^{-1} & T B \\
C T^{-1} & D
\end{array}\right]
$$

has controllability and observability Gramians given by

$$
Y=\left[\begin{array}{cccc}
\Sigma_{1} & & & \\
& \Sigma_{2} & & \\
& & 0 & \\
& & & 0
\end{array}\right]
$$

and

$$
X=\left[\begin{array}{llll}
\Sigma_{1} & & & \\
& 0 & & \\
& & \Sigma_{3} & \\
& & & 0
\end{array}\right] .
$$

respectively, with $\Sigma_{1}, \Sigma_{2}, \Sigma_{3}$ diagonal and positive definite.

Since the uncontrollable and unobservable modes of any system realization are not present in the corresponding system transfer function, we can truncate the associated states, corresponding to the zeros in $Y$ or $X$ above, and obtain an equivalent minimal realization which has both Gramians equal to $\Sigma_{1}$. The original realization $M$ is reducible in that there exists a lower order realization $M_{r}$ with associated operator $G_{r}$, such that $\left\|G-G_{r}\right\|_{\infty}=0$, which gives us the following corollary to Theorem 2 .

Corollary 3: Every stable 1-D system has a minimal realization which is balanced.

\section{B. Balanced I-D Model Reduction}

Consider a stable discrete-time system with the following realization:

$$
M=\left[\begin{array}{ccc}
A_{11} & A_{12} & B_{1} \\
A_{21} & A_{22} & B_{2} \\
C_{1} & C_{2} & D
\end{array}\right] .
$$

Suppose $Y$ and $X$ are two positive semidefinite symmetric matrices satisfying the following Lyapunov inequalities:

$$
\begin{aligned}
& A Y A^{*}-Y+B B^{*} \leq 0 \\
& A^{*} X A-X+C^{*} C \leq 0 .
\end{aligned}
$$

We have replaced the equalities in (4) and (5) with inequalities in (6) and (7) to generalize the 1-D system results to uncertain systems. The significance of these inequalities in the 1-D case is that while the zero-valued eigenvalues of $Y$ or $X$ still have corresponding uncontrollable and/or unobservable states, the converse need not be true. This is most easily seen via a simple example: consider the system with realization

$$
\begin{aligned}
& A=\left[\begin{array}{cc}
0.5 & 0 \\
0 & 0.2
\end{array}\right] \\
& B=\left[\begin{array}{l}
1 \\
0
\end{array}\right] \\
& C=\left[\begin{array}{ll}
0 & 1
\end{array}\right] .
\end{aligned}
$$

It is clear that

$$
Y=\left[\begin{array}{ll}
2 & 0 \\
0 & 0
\end{array}\right]
$$

and

$$
X=\left[\begin{array}{ll}
0 & 0 \\
0 & 2
\end{array}\right]
$$


satisfy the inequalities of (6) and (7), as the first state is unobservable and the second state is uncontrollable. However

$$
\begin{aligned}
Y & =X \\
& =\left[\begin{array}{ll}
2 & 0 \\
0 & 2
\end{array}\right]
\end{aligned}
$$

for example, also satisfy the inequalities of (6) and (7). Thus, we can truncate states as suggested by Corollary 3 and balance $X$ and $Y$ exactly as before, but the resulting system may not be minimal. Subsequently, when we refer to balanced system realizations it will be in this looser sense, that is, with $Y>0$ and $X>0$ satisfying the Lyapunov inequalities above and

$$
\begin{aligned}
Y & =X \\
& =\left[\begin{array}{cc}
\Sigma_{1} & 0 \\
0 & \Sigma_{2}
\end{array}\right]
\end{aligned}
$$

with

$$
\begin{aligned}
& \Sigma_{1}=\operatorname{diag}\left(\sigma_{1} I_{s_{1}}, \sigma_{2} I_{s_{2}}, \cdots, \sigma_{r} I_{s_{r}}\right)>0 \\
& \Sigma_{2}=\operatorname{diag}\left(\sigma_{r+1} I_{s_{r+1}}, \sigma_{r+2} I_{s_{r+2}}, \cdots, \sigma_{n} I_{s_{r_{n}}}\right)>0
\end{aligned}
$$

where $s_{i}$ denotes the multiplicity of $\sigma_{i}$. Note that the $\sigma_{i}$ are not necessarily ordered and are assumed to be distinct, although distinctness is not required (see the remark at end of the section).

The balanced truncation model reduction results, first given for 1-D discrete-time systems in [21] and for multidimensional or uncertain systems in [32], are now stated. The results are separated into a lemma stating that the truncation of a stable, balanced realization is also stable and balanced and a theorem stating the upper error bound results, measured in the $H_{\infty}$ norm. The proof for the lemma can be found in [21]. A proof for the theorem is given in the Appendix; this proof generalizes immediately to system representations which include uncertainty.

Lemma 4: Suppose

$$
M=\left[\begin{array}{ccc}
A_{11} & A_{12} & B_{1} \\
A_{21} & A_{22} & B_{2} \\
C_{1} & C_{2} & D
\end{array}\right]
$$

is a balanced, stable realization. Then the truncated system realization given by

$$
M_{r}=\left[\begin{array}{cc}
A_{11} & B_{1} \\
C_{1} & D
\end{array}\right]
$$

is also balanced and stable.

Theorem 5: Suppose

$$
M=\left[\begin{array}{ccc}
A_{11} & A_{12} & B_{1} \\
A_{21} & A_{22} & B_{2} \\
C_{1} & C_{2} & D
\end{array}\right]
$$

is a balanced, stable realization for $G$ with

$$
\begin{aligned}
X & =Y \\
& =\left[\begin{array}{cc}
\Sigma_{1} & 0 \\
0 & \Sigma_{2}
\end{array}\right]>0
\end{aligned}
$$

as defined in (8). Let

$$
M_{r}=\left[\begin{array}{cc}
A_{11} & B_{1} \\
C_{1} & D
\end{array}\right]
$$

denote the balanced, stable, truncated system realization for $G_{r}$. Then

$$
\left\|G-G_{r}\right\|_{\infty} \leq 2 \sum_{i=r+1}^{n} \sigma_{i} .
$$

Proof: See the Appendix.

Remark 6: Distinctness of the $\sigma_{i}$ in $\Sigma_{1}$ and $\Sigma_{2}$ is not required for stability of the truncated subsystem in the discretetime case, although it is in the continuous-time case. However, there is no reason to truncate the system in the middle of a $\sigma_{i} I_{s_{i}}$ block as this will not improve the error bound.

For 1-D system realizations with no uncertainty the role of the system Lyapunov equations, and of the associated controllability and observability Gramians, in balanced truncation model reduction and in terms of quantifying system minimality are well defined. In the next section, we consider these concepts for system realizations which incorporate uncertainty descriptions into the model definitions.

\section{SYSTEM REPRESENTATIONS WITH UNCERTAINTY}

We now define and discuss stable realizations and balanced model reduction methods for uncertain systems. Guaranteed error bounds are stated and proven for these reduction methods. We assume throughout this section that the uncertainty structure is defined as in (1) with one of the $\delta_{i}$ representing the shift operator and the remaining $\delta_{i}$ representing arbitrary LTV operators on $l_{2}$, unless otherwise noted.

\section{A. Balanced System Realizations}

Consider the system in Fig. 1 , where $\Delta \in \Delta$ is defined as in (1) and $M$ is the representation matrix. Such a system is stable when the I/O map $(\Delta \star M)$ is well posed for every $\Delta \in \mathbf{B}_{\Delta}$, that is, when $(I-A \Delta)$ is invertible as an operator on $l_{2}$ for every $\Delta \in \mathbf{B}_{\Delta}$. Using this invertibility condition as the basic conceptual definition for stability of uncertain systems, an easily computed LMI-based stability condition can be constructed; this has been proven in [29] and is also stated below in Theorem 8 . This stability result is an extension of the results on robust stability of systems with time-varying uncertainty given in [24] and concurrently in [31] and is proven via methods similar to those in [24]. Details can be found in [29]. The uncertainty structure $\hat{\Delta}$ referred to in Theorem 8 is more general than that defined in (1) in that full blocks are also allowed, that is

$$
\begin{gathered}
\hat{\Delta}=\left\{\operatorname{diag}\left[\Delta, \Delta_{1}, \cdots, \Delta_{F}\right]: \Delta \in \Delta, \Delta_{j} \in \mathcal{L}\left(l_{2}^{m_{j}}\right),\right. \\
j=1, \cdots, F\}
\end{gathered}
$$

where $m_{j}$ denotes the dimension of the domain of $\Delta_{j}$, and we assume all $\Delta_{j}$ are time-varying operators.

Definition 7: Let $n=\sum_{i=1}^{p} n_{i}+\sum_{j=1}^{F} m_{j}$. The commutative matrix set for a given uncertainty set $\boldsymbol{\Delta}$ is denoted by $\mathcal{T}$ and defined by

$$
\mathcal{T}=\left\{T \in \mathbb{C}^{n \times n}: T \Delta=\Delta T, \text { for all } \Delta \in \Delta\right\} .
$$

For $\hat{\boldsymbol{\Delta}}$ as defined in (9), $T \in \mathcal{T}$ if $T=\operatorname{diag}\left[T_{1}\right.$, $\left.\cdots, T_{p}, t_{1} I_{m_{1}}, \cdots, t_{F} I_{m_{F}}\right]$, where each $T_{i} \in \mathbb{C}^{n_{i} \times n_{i}}$ and $t_{j} \in \mathbb{C}$. 
Theorem 8: Given an uncertainty set $\hat{\Delta} \subset \mathcal{L}\left(l_{2}\right)$, and a constant matrix $A$, then there exists a matrix $Y>0, Y \in \mathcal{T}$ such that

$$
A Y A^{*}-Y<0
$$

if and only if

$$
(I-A \hat{\Delta}) \text { is invertible in } \mathcal{L}\left(l_{2}\right), \text { for all } \hat{\Delta} \in \mathbf{B}_{\hat{\Delta}}
$$

The existence of a matrix $X>0, X \in \mathcal{T}$ satisfying $A^{*} X A-X<0$ is clearly also equivalent to the invertibility of the operator $(I-A \hat{\Delta})$, for all $\hat{\Delta} \in \mathbf{B}_{\hat{\Delta}}$. Note that the feasibility of these LMI's in $Y$ and $X$ is also equivalent to the maximum singular value condition

$$
\text { there exists } T \in \mathcal{T}: \bar{\sigma}\left(T A T^{-1}\right)<1 \text {. }
$$

By scaling $Y$ and $X$ one can immediately deduce the following lemma.

Lemma 9: $(\boldsymbol{\Delta}, M)$ is stable if and only if there exist $Y>0$ and $X>0$, both in $\mathcal{T}$, which satisfy

$$
A Y A^{*}-Y+B B^{*}<0
$$

and

$$
A^{*} X A-X+C^{*} C<0 .
$$

As in the 1-D case, we can use nonstrict inequalities in (10). Stability of $(\boldsymbol{\Delta}, M)$ then implies that there exist $Y \geq 0$ and $X \geq 0$ both in $\mathcal{T}$ such that

$$
A Y A^{*}-Y+B B^{*} \leq 0
$$

and

$$
A^{*} X A-X+C^{*} C \leq 0 .
$$

We refer to the nonunique matrices $Y$ and $X$ which satisfy (11) as generalized Gramians. We can show that if there exist singular generalized Gramians, then the uncertain system realization may be reduced with no error (see [3] and [6]). As a result, in deriving the model reduction error bounds, we need only consider the case of strict inequalities.

To define a notion of balanced realizations for uncertain systems, we proceed as in the 1-D case by defining a similarity transformation as an invertible matrix, $T$, which transforms the system states such that the realization $M$ is transformed to $\hat{M}$ as in Section III-A. We consider similarity transformations in $\mathcal{T}$ for which it can readily be shown that

$$
(\Delta \star M)=(\Delta \star \hat{M}), \quad \forall \Delta \in \Delta .
$$

Note that when $T \in T, \hat{Y}=T Y T^{*}$ and $\hat{X}=\left(T^{-1}\right)^{*} X T^{-1}$ are solutions to the Lyapunov inequalities for $\hat{M}$. Thus we define balanced realizations for uncertain systems as follows.

Definition 10: An uncertain system realization, $(\Delta, M)$, is balanced if there exist $Y>0$ and $X>0$, both in $\mathcal{T}$, that satisfy the inequalities in (10) and

$$
\begin{aligned}
Y & =X \\
& =\Sigma \\
& =\operatorname{diag}\left[\Sigma_{1}, \cdots, \Sigma_{p}\right]
\end{aligned}
$$

where $\Sigma_{i}>0$ are diagonal, and $\operatorname{dim}\left(\Sigma_{i}\right)=\operatorname{dim}\left(I_{n_{i}}\right)$.
For stable uncertain systems, the existence of balanced realizations is guaranteed by Lemma 9 and by (12). Neither the balanced realization nor the balanced $\Sigma$ is unique.

Remark 11: Note that permutations do not affect stability or sign-definiteness of the Lyapunov inequalities. That is, let $\Pi$ be any matrix such that $\Pi \Pi^{T}=I$, and suppose we have a solution $P \in \mathcal{T}$ to (10). Denote $\mathcal{A}=\Pi^{T} A \Pi, \mathcal{B}=\Pi^{T} B \Pi$, and $\mathcal{P}=\Pi^{T} P \Pi$. Then

$$
\mathcal{A P} \mathcal{A}^{*}-\mathcal{P}+\mathcal{B} B^{*}=\Pi^{T}\left(A P A^{*}-P+B B^{*}\right) \Pi<0 .
$$

\section{B. Error Bounds: Balanced Truncation Model Reduction}

To quantify the error resulting from reducing uncertain systems, we use the structured induced 2-norm, (SI2-norm) which we define as follows.

Definition 12: The SI2 norm of a stable system $(\Delta, M)$ is given by

$$
\|\Delta \star M\|_{\text {SI2 }}=\sup _{\Delta \in \mathrm{B}_{\Delta}}\|\Delta \star M\|_{l_{2} \rightarrow l_{2}}
$$

Note that the SI2-norm is a generalization of the $H_{\infty}$ norm, and for 1-D system realizations with no uncertainty these norms are identical.

The difference between two realizations, $\left(\boldsymbol{\Delta}_{1}, M_{1}\right)$ and $\left(\Delta_{2}, M_{2}\right)$, is evaluated in the SI2-norm by forming the difference realization of $(\tilde{\Delta} \star \tilde{E})=\left(\Delta_{1} \star M_{1}\right)-\left(\Delta_{2} \star M_{2}\right)$, that is, by setting

$$
\begin{aligned}
& \tilde{E}=\left[\begin{array}{ccc}
A_{1} & 0 & B_{1} \\
0 & A_{2} & B_{2} \\
C_{1} & -C_{2} & D_{1}-D_{2}
\end{array}\right] \\
& \tilde{\Delta}=\left[\begin{array}{cc}
\Delta_{1} & 0 \\
0 & \Delta_{2}
\end{array}\right]
\end{aligned}
$$

and computing $\|\tilde{\Delta} \star \tilde{E}\|_{\mathrm{SI} 2}$.

An equivalent formulation for the SI2-norm of a system with $\Delta$ representing LTV uncertainty is given in the following lemma. This formulation more readily allows for computation via recent software packages developed for solving LMI's ([16] and [17]).

Lemma 13: The SI2-norm of a stable system $(\boldsymbol{\Delta}, M)$ is given by

$$
\begin{aligned}
& \|\Delta \star M\|_{\text {SI2 }}=\inf \{\gamma: \text { there exists } T \text { such that } \\
& \left.\bar{\sigma}\left(\left[\begin{array}{cc}
T A T^{-1} & \frac{1}{\gamma^{1 / 2}} T B \\
\frac{1}{\gamma^{1 / 2}} C T^{-1} & \frac{1}{\gamma} D
\end{array}\right]\right)<1\right\}
\end{aligned}
$$

where $T \in \mathcal{T}$ and $\gamma>0$.

Proof: Let $\Delta_{F} \in \mathcal{L}\left(l_{2}^{p}\right)$ and

$$
M_{\gamma}=\left[\begin{array}{cc}
A & \frac{1}{\gamma^{1 / 2}} B \\
\frac{1}{\gamma^{1 / 2}} C & \frac{1}{\gamma} D
\end{array}\right] .
$$


Then for a stable system $(\Delta, M)$

$$
\begin{aligned}
& \inf \{\gamma: \text { there exists } T \text { such that } \\
& \left.\quad=\left(\left[\begin{array}{cc}
T A T^{-1} & \frac{1}{\gamma^{1 / 2}} T B \\
\frac{1}{\gamma^{1 / 2}} C T^{-1} & \frac{1}{\gamma} D
\end{array}\right]\right)<1\right\} \\
& \quad=\inf \{\gamma: \text { there exists } Y>0 \text { such that } \\
& \left.M_{\gamma}\left[\begin{array}{ll}
Y & 0 \\
0 & I
\end{array}\right] M_{\gamma}^{*}-\left[\begin{array}{cc}
Y & 0 \\
0 & I
\end{array}\right]<0\right\}
\end{aligned}
$$

for $Y$ and $T$ both in $\mathcal{T}$ and $\gamma>0$. Applying Theorem 8 and the stability assumption on $(\Delta, M)$, we see that $(17)$ is equivalent to

$$
\begin{aligned}
& \inf \left\{\gamma:\left(I-M_{\gamma}\left[\begin{array}{cc}
\Delta & 0 \\
0 & \Delta_{F}
\end{array}\right]\right)\right. \\
& \text { is invertible in } \left.\mathcal{L}\left(l_{2}\right) \text { for all } \Delta_{F} \in \mathbf{B}_{\Delta_{F}}\right\} \\
& \quad=\inf \left\{\gamma:\left[\begin{array}{cc}
I-A \Delta & -\frac{1}{\gamma^{1 / 2}} B \Delta_{F} \\
0 & I-\left(\frac{1}{\gamma} \Delta \star M\right) \Delta_{F}
\end{array}\right]\right. \\
& \text { is invertible in } \left.\mathcal{L}\left(l_{2}\right) \text { for all } \Delta_{F} \in \mathbf{B}_{\Delta_{F}}\right\} \\
& \quad=\inf \left\{\gamma:\left[I-\left(\frac{1}{\gamma} \Delta \star M\right) \Delta_{F}\right]\right. \\
& \text { is invertible in } \left.\mathcal{L}\left(l_{2}\right) \text { for all } \Delta_{F} \in \mathbf{B}_{\Delta_{F}}\right\} \\
& \quad=\sup _{\Delta \in \mathbf{B}_{\Delta}}\|\Delta \star M\|_{l_{2} \rightarrow l_{2} .}
\end{aligned}
$$

Using Lemma 13, we can immediately prove the following result which generalizes Lemma 22 (see the Appendix) to uncertain systems.

Lemma 14: Suppose $(\boldsymbol{\Delta}, M)$ represents a stable uncertain system, then $\|\boldsymbol{\Delta} \star M\|_{\mathrm{SI} 2} \leq 1$ if and only if there is a realization $\hat{M}$, where $(\Delta \star \bar{M})=(\Delta \star \hat{M})$ for all $\Delta \in \Delta$ such that $\bar{\sigma}(\hat{M}) \leq 1$.

A constant matrix $X$ is said to be contractive if $\|X\|=$ $\bar{\sigma}(X) \leq 1$ and strictly contractive if $\|X\|<1$. If we consider uncertainty structures which contain linear time-invariant (LTI) or commuting operators, for example, real parametric uncertainty, LTI dynamic uncertainty, or when the $\Delta$ set represents multiple shift operators corresponding to different transform variables in a multidimensional system, then the expression on the right in (16) is an upper bound for the system SI2norm. Therefore, the existence of a contractive realization is a sufficient condition for $\|\Delta \star M\|_{\text {SI2 }} \leq 1$ when the $\delta_{i}$ of the $\Delta$ block are LTI operators. This sufficiency is all that is needed for the balanced truncation model reduction bounds to hold for both multidimensional and uncertain systems.
To derive the model reduction error bounds for balanced uncertain systems, we partition the system matrices $A, B, C$, and $\Sigma$ so as to separate the subblocks which will be truncated. That is, $A, B$, and $C$ are partitioned compatibly with the block structure $\Delta$ as

$$
\begin{aligned}
A & =\left[\begin{array}{ccc}
A_{11} & \cdots & A_{1 p} \\
\vdots & \ddots & \vdots \\
A_{p 1} & \cdots & A_{p p}
\end{array}\right] \\
B & =\left[\begin{array}{c}
B_{1} \\
\vdots \\
B_{p}
\end{array}\right] \\
C & =\left[\begin{array}{lll}
C_{1} & \cdots & C_{p}
\end{array}\right] .
\end{aligned}
$$

We further partition each block of $\Sigma$ by $\Sigma_{i}=\operatorname{diag}\left[\hat{\Sigma}_{1 i}, \Sigma_{2 i}\right]$, for $i=1, \cdots, p$, where the realization submatrices corresponding to $\Sigma_{2 i}$ will be truncated. Denote

$$
\begin{aligned}
\hat{\Sigma}_{1 i} & =\operatorname{diag}\left[\sigma_{i 1} I_{s_{i 1}}, \cdots, \sigma_{i k_{i}} I_{s_{i k_{i}}}\right] \\
\text { and } & \\
\Sigma_{2 i} & =\operatorname{diag}\left[\sigma_{i\left(k_{i}+1\right)} I_{s_{i\left(k_{i}+1\right)}}, \cdots, \sigma_{i t_{i}} I_{s_{i t_{i}}}\right] \quad k_{i} \leq t_{i} .
\end{aligned}
$$

We then truncate both $\Sigma_{2 i}$ and the corresponding parameter matrices, for example, we truncate

and

$$
\begin{aligned}
A_{11} & =\left[\begin{array}{ll}
\hat{A}_{11} & A_{11_{12}} \\
A_{11_{21}} & A_{11_{22}}
\end{array}\right] \\
B_{1} & =\left[\begin{array}{l}
\hat{B}_{1} \\
B_{1_{2}}
\end{array}\right]
\end{aligned}
$$

$$
C_{1}=\left[\begin{array}{ll}
\hat{C}_{1} & C_{1_{2}}
\end{array}\right]
$$

to $\hat{A}_{11}, \hat{B}_{1}$, and $\hat{C}_{1}$. Partitioning and truncating each $A_{i j}, B_{j}$, and $C_{i}, i, j=1, \cdots, p$ similarly results in the following truncated system:

$$
\begin{aligned}
M_{r} & =\left[\begin{array}{cc}
\hat{A} & \hat{B} \\
\hat{C} & D
\end{array}\right] \\
& =\left[\begin{array}{cccc}
\hat{A}_{11} & \cdots & \hat{A}_{1 p} & \hat{B}_{1} \\
\vdots & \ddots & \vdots & \vdots \\
\hat{A}_{p 1} & \cdots & \hat{\hat{A}}_{p} & \hat{B}_{p} \\
\hat{C}_{1} & \cdots & \hat{C}_{p} & D
\end{array}\right]
\end{aligned}
$$

with uncertainty set $\boldsymbol{\Delta}_{r}=\left\{\operatorname{diag}\left[\delta_{1} I_{r_{1}}, \cdots, \delta_{p} I_{r_{p}}\right]: \delta_{i} \in\right.$ $\left.\mathcal{L}\left(l_{2}\right)\right\}$ where $r_{i}=\sum_{j=1}^{k_{i}} s_{i j}$. Note that $\boldsymbol{\Delta}_{r}$ is constructed with the same uncertainty variables $\delta_{i}$ as is $\boldsymbol{\Delta}$, thus the $\Delta \in \Delta$ and $\Delta_{r} \in \Delta_{r}$ are not independent.

As in the 1-D case, truncating a balanced stable uncertain system realization results in a lower dimension realization that is balanced and stable which is easily seen by considering the system Lyapunov inequalities. We now state the balanced truncation model reduction error bound theorem for uncertain and multidimensional systems.

Theorem 15: Suppose $\left(\boldsymbol{\Delta}_{r}, M_{r}\right)$ is the reduced model obtained from the balanced stable system $(\Delta, M)$. Then

$$
\left\|(\boldsymbol{\Delta} \star M)-\left(\boldsymbol{\Delta}_{r} \star M_{r}\right)\right\|_{\mathrm{SI} 2} \leq 2 \sum_{i=1}^{p} \sum_{j=k_{i}+1}^{t_{i}} \sigma_{i j} .
$$


Proof: See the Appendix.

\section{IMPROVED ERROR BOUNDS: LMI-BASED MODEL REDUCTION}

A tighter model reduction bound than that given in Theorem 15 can be achieved using the solutions to the system Lyapunov inequalities by utilizing machinery presented by Packard et al. [27], [28]; similar machinery is also given in [2] and [22]. The tighter error bound is derived from Lemma 13 and from the results of [27] and [28] which are stated below. We first define some notation.

Throughout this section we refer to the following uncertainty structures:

$$
\begin{aligned}
\boldsymbol{\Delta} & =\left\{\operatorname{diag}\left[\delta_{1} I_{n_{1}}, \delta_{2} I_{n_{2}}, \cdots, \delta_{p} I_{n_{p}}\right]: \delta_{i} \in \mathcal{L}\left(l_{2}\right)\right\} \\
\boldsymbol{\Delta}_{r} & =\mathcal{I}_{r}(\boldsymbol{\Delta}) \\
& =\left\{\operatorname{diag}\left[\delta_{1} I_{r_{1}}, \delta_{2} I_{r_{2}}, \cdots, \delta_{p} I_{r_{p}}\right]: \delta_{i} \in \mathcal{L}\left(l_{2}\right)\right\}
\end{aligned}
$$

where the notation $\mathcal{I}_{r}(\Delta)$ is used to emphasize that $\Delta_{r}$ represents a reduced- or lower-dimension copy of $\Delta$, and is not an independent uncertainty structure; also

$$
\tilde{\boldsymbol{\Delta}}=\left\{\left[\begin{array}{cc}
\Delta & 0 \\
0 & \Delta_{r}
\end{array}\right]: \Delta \in \boldsymbol{\Delta}, \Delta_{r} \in \mathcal{I}_{r}(\boldsymbol{\Delta})\right\} .
$$

We again assume one of the $\delta_{i}$ represents the shift operator on $l_{2}$, and the remaining $\delta_{i}$ are LTV operators on $l_{2}$. The commutative matrix sets corresponding to these uncertainty structures are denoted by $\mathcal{T}, \mathcal{T}_{r}$, and $\tilde{\mathcal{T}}$. Note that as a result of the dependence between $\Delta$ and $\Delta_{T}$, the set $\tilde{\mathcal{T}}$ for $\tilde{\Delta}$ includes matrices with the following block structure:

$$
\tilde{T}=\left[\begin{array}{cc}
\operatorname{diag}\left(T_{i}^{n}\right) & \operatorname{diag}\left(T_{i}^{n r}\right) \\
\operatorname{diag}\left(T_{i}^{r n}\right) & \operatorname{diag}\left(T_{i}^{r}\right)
\end{array}\right]
$$

where $\operatorname{dim}\left(T_{i}^{n}\right)=n_{i} \times n_{i}, \operatorname{dim}\left(T_{i}^{n r}\right)=n_{i} \times r_{i}, \operatorname{dim}\left(T_{i}^{r n}\right)=$ $r_{i} \times n_{i}$ and $\operatorname{dim}\left(T_{i}^{r}\right)=r_{i} \times r_{i}$ for all $i=1, \cdots, p$. For the uncertain system representations $(\Delta, M)$ and $\left(\Delta_{r}, M_{r}\right)$, the difference system $(\tilde{\Delta} \star \tilde{E})=(\Delta \star M)-\left(\Delta_{r} \star M_{r}\right)$ is formed as in (15).

Given a system representation $(\Delta, M)$, we show that for any $\epsilon>0$, a lower-order realization $\left(\boldsymbol{\Delta}_{r}, M_{r}\right)$ exists such that the SI2-norm of the difference system $(\tilde{\boldsymbol{\Delta}}, \tilde{E})$ is bounded above by $\epsilon$ if and only if there exist solutions, $X_{\epsilon}$ and $Y_{\epsilon}$, to the Lyapunov inequalities (10) that also satisfy a rank constraint.

Theorem 16: Given a system realization $M=\left[\begin{array}{cc}A & B \\ C\end{array}\right]$ with uncertainty structure $\boldsymbol{\Delta}$, then there exist $M_{r}=\left[\begin{array}{cc}A_{r} & B_{r} \\ C_{r} & D_{r}\end{array}\right]$ and $\boldsymbol{\Delta}_{r}$ such that $\|\tilde{\boldsymbol{\Delta}} \star \tilde{E}\|_{\text {SI2 }} \leq \epsilon$ if and only if there exists $X_{\epsilon}>0$ and $Y_{\epsilon}>0$, both in $\mathcal{T}$, satisfying:

i) $A X_{\epsilon} A^{*}-X_{\epsilon}+B B^{*}<0$;

ii) $A^{*} Y_{\epsilon} A-Y_{\epsilon}+C^{*} C<0$;

iii) $\lambda_{\min }\left(X_{\epsilon} Y_{\epsilon}\right)=\epsilon^{2}$, with multiplicity $\sum_{i=1}^{p}\left(n_{i}-r_{i}\right)$

where $\epsilon>0$.

The existence of solutions, $X_{\epsilon}>0$ and $Y_{\epsilon}>0$, to the Lyapunov inequalities is both necessary and sufficient for obtaining the bound of $\epsilon$. Furthermore, a related necessary and sufficient condition also holds for $\epsilon=0$; see [3] and [6] for details, as well as Remark 20. Thus, this error bound is tight in the sense that if we can find optimal solutions to the Lyapunov inequalities, that is to a pair of LMI's, we will be able to find a lower-order approximation to the full order model that results in the least possible error. Applying Theorem 16 recursively to a balanced realization results in error bounds for model reduction which are lower than those of Theorem 15 by a factor of two; however, the resulting additive error bounds are not tight.

To prove Theorem 16, we use the following linear algebra results, Lemmas 17 and 18, taken directly from [27] and [28]; proofs may be found in [27]. These results are applied to the error system $(\tilde{\Delta}, \tilde{E})$, in conjunction with Lemma 13 , to construct the LMI conditions i) and ii) and the rank constraint iii) of Theorem 16. Let $R \in \mathbb{R}^{l \times l}, U \in \mathbb{R}^{l \times m}$, and $V \in \mathbb{R}^{q \times l}$, where $m, q \leq l$.

Lemma 17 [27]: Suppose $U_{\perp} \in \mathbb{R}^{l \times(l-m)}$ and $V_{\perp} \in$ $\mathbb{R}^{(l-q) \times l}$ satisfy $U^{*} U_{\perp}=0, V V_{\perp}^{*}=0$, with $\left[\begin{array}{ll}U & U_{\perp}\end{array}\right]$ and $\left[\begin{array}{l}V \\ V_{\perp}\end{array}\right]$ invertible. Let $\mathcal{Z} \in \mathbb{C}^{l \times l}$ be a given set of positive definite Hermitian matrices. Then

$$
\inf _{\substack{Q \in \mathbb{R}^{m \times q} \\ Z \in \mathcal{Z}}} \bar{\sigma}\left[Z^{1 / 2}(R+U Q V) Z^{-1 / 2}\right]<1
$$

if and only if there exists $Z \in \mathcal{Z}$ such that

$$
V_{\perp}\left(R^{*} Z R-Z\right) V_{-}^{*}<0
$$

and

$$
U_{\perp}^{*}\left(R Z^{-1} R^{*}-Z^{-1}\right) U_{\perp}<0 .
$$

The LMI conditions given in (21) are, respectively, convex in $Z$ and $Z^{-1}$, but the two conditions together cannot be formulated as a convex constraint on either variable for a general set of matrices $\mathcal{Z}$. However, for the model reduction problem addressed in this paper (as for the synthesis problem discussed in [28]) these two conditions can be reformulated into one jointly convex condition, coupled by a nonconvex rank constraint (see Section VI for a brief discussion of computational issues).

Using the notation of [28] for our model reduction problem, we define

$$
\begin{aligned}
R & =\left[\begin{array}{lll}
A & 0 & B \\
0 & 0 & 0 \\
C & 0 & D
\end{array}\right] \\
U & =\left[\begin{array}{ll}
0 & 0 \\
I & 0 \\
0 & I
\end{array}\right] \\
V & =\left[\begin{array}{lll}
0 & I & 0 \\
0 & 0 & I
\end{array}\right]
\end{aligned}
$$

and

$$
Q=\left[\begin{array}{rr}
A_{r} & B_{r} \\
-C_{r} & -D_{r}
\end{array}\right]
$$

Note that $M_{r}=\left[\begin{array}{cc}I & 0 \\ 0 & -I\end{array}\right] Q$ and $\tilde{E}=R+U Q V$; furthermore, $U_{\perp}=\left[\begin{array}{lll}I & 0 & 0\end{array}\right]^{T}$ and $V_{\perp}=\left[\begin{array}{lll}I & 0 & 0\end{array}\right]$. We then accordingly define $\tilde{U}=\left[\begin{array}{ll}U_{\perp, 1}^{T} & U_{\perp, 3}^{T}\end{array}\right]^{T}=\left[\begin{array}{ll}I & 0\end{array}\right]^{T}$ and $\tilde{V}=\left[\begin{array}{ll}V_{\perp, 1} & V_{\perp, 3}\end{array}\right]=\left[\begin{array}{ll}I & 0\end{array}\right]$ and note that the dimensions $m, q$, and $l$ correspond to the row and column dimensions of $M_{r}$ and the dimensions of $R$, respectively. 
In applying Lemma 17, if we set

$$
\mathcal{Z}:=\left\{\left[\begin{array}{cc}
\tilde{T} & 0 \\
0 & I
\end{array}\right]: \tilde{T} \in \tilde{T}, \tilde{T}=\tilde{T}^{*}>0\right\}
$$

then instead of casting (21) as a pair of convex constraints on $Y=\tilde{T}$ and $X=Y^{-1}$, we need only consider constraints on the submatrices of $X$ and $Y, X_{i}^{n}$, and $Y_{i}^{n}$, due to the structure of the matrices $R, U, V$, and $\tilde{T} \in \tilde{\mathcal{T}}$. In particular, the following lemma may be used to form one LMI constraint on $X_{i}^{n}$ and $Y_{i}^{n}$.

Lemma 18 [27]: Suppose $X \in \mathbb{C}^{n \times n}$ and $Y \in \mathbb{C}^{n \times n}$ with $X=X^{*}>0$ and $Y=Y^{*}>0$ given. Then there exist matrices $X_{2} \in \mathbb{C}^{n \times r}$ and $X_{3} \in \mathbb{C}^{r \times r}$, with $X_{3}=X_{3}^{*}>0$, such that

and

$$
\left[\begin{array}{ll}
X & X_{2} \\
X_{2}^{*} & X_{3}
\end{array}\right]>0
$$

$$
\left[\begin{array}{cc}
X & X_{2} \\
X_{2}^{*} & X_{3}
\end{array}\right]^{-1}=\left[\begin{array}{cc}
Y & Y_{12} \\
Y_{21} & Y_{3}
\end{array}\right]
$$

if and only if

$$
\left[\begin{array}{ll}
X & I \\
I & Y
\end{array}\right] \geq 0
$$

and

$$
\operatorname{rank}\left[\begin{array}{cc}
X & I \\
I & Y
\end{array}\right] \leq n+r
$$

In the model reduction framework we consider, the dimensions $n$ and $r$ in Lemma 18 correspond to the dimensions of the uncertainty structures $\Delta$ and $\Delta_{r}$; that is, $n=\sum_{i=1}^{p} n_{i}$ and $r=\sum_{i=1}^{p} r_{i}$. The following lemma is a direct result of the preceding lemmas and is also a corollary to [28, Th. 6.3].

Lemma 19: Suppose the realization $(\Delta, M)$ is given, with $R, U, V, \tilde{U}$, and $\tilde{V}$ defined as in (22). Then there exists a realization $\left(\boldsymbol{\Delta}_{r}, M_{r}\right)$ and a matrix $Z \in \tilde{\mathcal{T}}, Z>0$ satisfying

$$
\bar{\sigma}\left(\left[\begin{array}{ll}
Z & 0 \\
0 & I
\end{array}\right] \tilde{E}\left[\begin{array}{cc}
Z^{-1} & 0 \\
0 & I
\end{array}\right]\right)<1
$$

if and only if there exist $X_{i}^{n}>0, Y_{i}^{n}>0$ for $i=1, \cdots, p$ satisfying:

$$
\begin{array}{ll}
\text { a) } & \tilde{U}^{T}\left(M\left[\begin{array}{cc}
\operatorname{diag}\left(X_{i}^{n}\right) & 0 \\
0 & I
\end{array}\right] M^{T}-\left[\begin{array}{cc}
\operatorname{diag}\left(X_{i}^{n}\right) & 0 \\
0 & I
\end{array}\right]\right) \tilde{U} \\
& <0 \\
\text { b) } & \tilde{V}\left(M^{T}\left[\begin{array}{cc}
\operatorname{diag}\left(Y_{i}^{n}\right) & 0 \\
0 & I
\end{array}\right] M-\left[\begin{array}{cc}
\operatorname{diag}\left(Y_{i}^{n}\right) & 0 \\
0 & I
\end{array}\right]\right) \tilde{V}^{T} \\
& <0 \\
\text { c) }\left[\begin{array}{cc}
X_{i}^{n} & I \\
I & Y_{i}^{n}
\end{array}\right] \geq 0 .
\end{array}
$$

If these conditions are feasible, then the dimensions of $\boldsymbol{\Delta}_{r}$ and $M_{r}$ are determined by defining $r_{i}=\operatorname{rank}\left[X_{i}^{n}-\left(Y_{i}^{n}\right)^{-1}\right]$ for each $i=1, \cdots, p$.

We can now prove Theorem 16 . For convenience, we denote the $\epsilon$-scaled difference system realization by

$$
\tilde{E}_{\epsilon}=\left[\begin{array}{ccc}
A & 0 & \frac{1}{\epsilon^{1 / 2}} B \\
0 & A_{r} & \frac{1}{\epsilon^{1 / 2}} B_{r} \\
\frac{1}{\epsilon^{1 / 2}} C & -\frac{1}{\epsilon^{1 / 2}} C_{r} & \frac{1}{\epsilon}\left(D-D_{r}\right)
\end{array}\right] .
$$

Proof: By Lemma 13, $\|\tilde{\Delta} \star \tilde{E}\|_{\text {S12 }} \leq \epsilon$ if and only if there exists $T \in \tilde{\mathcal{T}}$ such that

$$
\bar{\sigma}\left(\left[\begin{array}{cc}
T & 0 \\
0 & I
\end{array}\right] \tilde{E}_{\epsilon}\left[\begin{array}{cc}
T^{-1} & 0 \\
0 & I
\end{array}\right]\right)<1 .
$$

Now we need only apply Lemma 19 to $\tilde{E}_{\epsilon}$ and multiply out the matrices in statements a) and b) of Lemma 19. Then $\|\tilde{\boldsymbol{\Delta}} \star \tilde{E}\|_{\mathrm{SI} 2} \leq \epsilon$ if and only if there exist $X=\operatorname{diag}\left(X_{i}^{n}\right)>0$, and $Y=\operatorname{diag}\left(Y_{i}^{n}\right)>0$ for $i=1, \cdots, p$ satisfying

$$
\begin{gathered}
A X A^{*}+\frac{1}{\epsilon} B B^{*}-X<0 \\
A^{*} Y A+\frac{1}{\epsilon} C^{*} C-Y<0
\end{gathered}
$$

and

$$
\left[\begin{array}{cc}
X_{i}^{n} & I \\
I & Y_{i}^{n}
\end{array}\right] \geq 0
$$

Multiplying the matrix inequalities in (24) by $\epsilon$, and denoting $X_{\epsilon}=\epsilon X$ and $Y_{\epsilon}=\epsilon Y$ gives i) and ii). Additionally we have

with

$$
\left[\begin{array}{cc}
X_{\epsilon i}^{n} & \epsilon I \\
\epsilon I & Y_{\epsilon i}^{n}
\end{array}\right] \geq 0
$$

$$
\operatorname{rank}\left[\frac{1}{\epsilon^{2}} X_{\epsilon i}^{n}-\left(Y_{\epsilon i}^{n}\right)^{-1}\right]=r_{i} .
$$

Condition iii) is obtained by premultiplying and postmultiplying the matrix in $(25)$ by $\left[(1 / \epsilon) I-\left(Y_{\epsilon i}^{n}\right)^{-1}\right]$ and $\left[\begin{array}{c}(1 / \epsilon) I \\ -\left(Y^{n}\right)^{-1}\end{array}\right]$, respectively, giving

$$
\frac{1}{\epsilon^{2}} X_{\epsilon i}^{n}-\left(Y_{\epsilon i}^{n}\right)^{-1} \geq 0
$$

thus $X_{\epsilon i}^{n} Y_{\epsilon i}^{n} \geq \epsilon^{2} I$. Applying the rank condition implies $\operatorname{rank}\left(X_{\epsilon i}^{n} Y_{\epsilon i}^{n}-\epsilon^{2} I\right)=r_{i}$, thus $\lambda_{\min }\left(X_{\epsilon i}^{n} Y_{\epsilon i}^{n}\right)=\epsilon^{2}$ with multiplicity $n_{i}-r_{i}$, for all $i=1, \cdots, p$. Since $X_{\epsilon}$ and $Y_{\epsilon}$ are block diagonal compositions of $X_{\epsilon i}^{n}$ and $Y_{\epsilon i}^{n}$ the result follows.

When the uncertainty structure, $\Delta$, contains LTI operators $\delta_{i}$, as in the case of multidimensional system models, the existence of $X_{\epsilon}$ and $Y_{\epsilon}$ are sufficient to ensure $\|\tilde{\Delta} \star \tilde{E}\|_{\text {SI2 }} \leq$ $\epsilon$. For $1-\mathrm{D}$ continuous-time systems with no uncertainty, similar results have been obtained by Kavranoglu and Bettayeb [23] via an alternate method that requires simultaneously computing a pair of matrices $B_{0}$ and $C_{0}$ augmenting the system realization matrices $B$ and $C$ and solutions $X$ and $Y$ to the augmented Lyapunov equations such that $\lambda_{\min }(X Y)=\epsilon^{2}$ with multiplicity $n-r$. Related results have also been presented recently by Helmersson [20].

Remark 20: For the proof of the model reduction error bounds to hold for both 1-D and uncertain systems, it is assumed there exist positive definite solutions $Y$ and $X$, both in $\mathcal{T}$, to the Lyapunov inequalities, in which case a nonzero error bound results. Clearly, we would prefer to reduce the system realization with no error if possible. To obtain an exact reducibility condition for uncertain systems which is analogous to the notion of reducibility in the 1-D case, we have also proven the $\epsilon=0$ case of Theorem 16. Quite different methods are required to prove this exact reducibility result; furthermore, the $\epsilon=0$ case leads not only to a notion of minimal realizations for uncertain systems, but also to the consideration of related realization theory concepts. For this 
reason, a complete exposition of these results is given in a separate paper [5]; details may also be found in [3] and [6].

\section{CONCLUSIONS}

We have presented LMI-based techniques, with guaranteed upper error bounds, for reducing uncertain and multidimensional systems. The systems we consider are described by an LFT of a constant realization matrix $M$ on a structured uncertainty set $\Delta$ and are assumed to be stable for all LTV operators $\Delta \in \mathbf{B}_{\Delta}$. As in the 1-D case, when the realization under consideration is not stable, feasible LMI solutions to (11) cannot be found. However, coprime factor methods generalizing the algorithm discussed in [25] have been constructed which may be used for unstable LFT realizations; these results will be reported elsewhere.

The development of computational methods for finding feasible solutions to LMI's such as the Lyapunov inequalities we consider has recently been a rather popular research area in the control community and many efficient convex optimization algorithms already exist, in particular, [16] and [17]. Although we can easily find feasible solutions to these LMI's, searching for singular solutions, or the rank-constrained optimal solutions desired in Theorem 16, presents a nonconvex optimization problem. We can, however, optimize each of the generalized Gramians separately; for example, one approach we have used is to find generalized Gramians $X$ and $Y$ with the objective being to minimize the trace of each. This leads to solutions for which the value of $\sum_{i}^{n} \lambda_{i}(Y X)$ is lowered, and hence the values of the separate $\lambda_{i}(Y X) \mathrm{s}$ are also lowered, effectively reducing the amount of error in both the balanced truncation and LMI-based model reduction methods. Preliminary tests of this suboptimal algorithm have been completed using LMI--LAB [17] and have yielded quite reasonable results. An application of this method has also been completed; see [3] and [4] for further details. Alternative heuristic algorithms for solving minimum rank LMI problems may be found in [11] and [12].

Lower error bounds for model reduction of uncertain systems are currently being investigated.

\section{APPENDIX}

To prove that the balanced truncation error bounds of Theorem 5 and Theorem 15 hold, we require a number of preliminary lemmas. We begin by discussing contractive matrices and associated results which are of general use for both 1-D and uncertain systems.

\section{A. Contractive Realizations}

Definition 21: A matrix $X$ is contractive if $\|X\|=\bar{\sigma}(X) \leq$ 1 and strictly contractive if $\|X\|<1$.

If the matrix $X$ is a realization matrix, the following lemma gives a well-known result ([10], [14]) on the relationship between the $\mathcal{H}_{\infty}$ norm of a transfer matrix and realizations of the transfer matrix.

Lemma 22: Suppose $G \in \mathcal{R} \mathcal{H}_{\infty}$ represents a discrete-time transfer matrix, then $\|G\|_{\infty} \leq 1(<1)$ if and only if there is a realization for $G$, denoted by $M=\left[\begin{array}{ll}A & B \\ C & D\end{array}\right]$ such that $M$ is contractive (strictly contractive).

A generalized version of this lemma for uncertain systems is given in Section IV. Lemma 22 and the following lemma, which relates the contractiveness of a matrix to that of related submatrices, provide the main steps in the proof of the error bounds for balanced truncation model reduction.

Lemma 23: Suppose

$$
U=\left[\begin{array}{cc}
U_{11} & U_{12} \\
Z & U_{22}
\end{array}\right]
$$

and

$$
V=\left[\begin{array}{cc}
V_{11} & Z \\
V_{21} & V_{22}
\end{array}\right]
$$

are contractive (strictly contractive). Then

$$
M:=\left[\begin{array}{ccc}
0 & \frac{1}{\sqrt{2}} U_{11} & U_{12} \\
\frac{1}{\sqrt{2}} V_{11} & Z & \frac{1}{\sqrt{2}} U_{22} \\
V_{21} & \frac{1}{\sqrt{2}} V_{22} & 0
\end{array}\right]
$$

is also contractive (strictly contractive).

Proof: The result is easily proved by dilating $M$ to the following matrix:

$$
M_{d}:=\left[\begin{array}{ccccc}
0 & \frac{1}{\sqrt{2}} U_{11} & U_{12} & \vdots & \frac{1}{\sqrt{2}} U_{11} \\
\frac{1}{\sqrt{2}} V_{11} & Z & \frac{1}{\sqrt{2}} V_{22} & \vdots & 0 \\
V_{21} & \frac{1}{\sqrt{2}} V_{22} & 0 & \vdots & -\frac{1}{\sqrt{2}} V_{22} \\
\cdots & 0 & -\frac{1}{\sqrt{2}} U_{22} & \vdots & -Z
\end{array}\right]
$$

and noting that $M_{d}^{*} M_{d} \leq I$.

Given a balanced system realization with $\Sigma_{2}=I$, we can prove the following lemma.

Lemma 24: Given a balanced realization of $(\Delta, M)$ with

$$
\begin{aligned}
Y & =X \\
& =\left[\begin{array}{cc}
\Sigma_{1} & 0 \\
0 & I
\end{array}\right]>0
\end{aligned}
$$

satisfying the Lyapunov inequalities, then

$$
\begin{aligned}
& {\left[\begin{array}{ccc}
\Sigma_{1}^{-1 / 2} A_{12} & \Sigma_{1}^{-1 / 2} A_{11} \Sigma_{1}^{1 / 2} & \Sigma_{1}^{-1 / 2} B_{1} \\
A_{22} & A_{21} \Sigma_{1}^{1 / 2} & B_{2}
\end{array}\right]} \\
& {\left[\begin{array}{cc}
A_{21} \Sigma_{1}^{-1 / 2} & A_{22} \\
\Sigma_{1}^{1 / 2} A_{11} \Sigma_{1}^{-1 / 2} & \Sigma_{1}^{1 / 2} A_{12} \\
C_{1} \Sigma_{1}^{-1 / 2} & C_{2}
\end{array}\right]}
\end{aligned}
$$

and

are contractive.

Proof: Rewriting (6) and (7) gives

and

$$
\left[\begin{array}{ll}
A & B
\end{array}\right]\left[\begin{array}{ll}
Y & 0 \\
0 & I
\end{array}\right]\left[\begin{array}{l}
A^{*} \\
B^{*}
\end{array}\right] \leq Y
$$

$$
\left[A^{*} \quad C^{*}\right]\left[\begin{array}{cc}
X & 0 \\
0 & I
\end{array}\right]\left[\begin{array}{l}
A \\
C
\end{array}\right] \leq X
$$


Premultiplying and postmultiplying (26) by $Y^{-1 / 2}$ and (27) by $X^{-1 / 2}$ shows that the matrices

$$
\left[Y^{-1 / 2} A Y^{1 / 2} \quad Y^{-1 / 2} B\right] \text { and }\left[\begin{array}{c}
X^{1 / 2} A X^{-1 / 2} \\
C X^{-1 / 2}
\end{array}\right]
$$

are contractive. Substituting $\left[\begin{array}{cc}\Sigma_{1} & 0 \\ 0 & I\end{array}\right]$ for $Y$ and $X$ in (28), and permuting the resulting matrices, gives the desired result.

\section{B. Balanced Truncation Model Reduction Error Bounds}

We now prove Theorem 5.

Proof: The proof of Theorem 5 relies heavily on the preceding lemmas. We assume that $\Sigma_{2}=I$. In this case, we must show that

$$
\left\|G-G_{r}\right\|_{\infty} \leq 2
$$

The final result follows from scaling and applying this result recursively.

By Lemma 22, it suffices to show that there exists a realization for $\frac{1}{2}\left(G-G_{r}\right)$ which is contractive. One realization for $\frac{1}{2}\left(G-G_{r}\right)$ is given by

$$
M=\left[\begin{array}{cccc}
A_{11} & 0 & 0 & \frac{1}{\sqrt{2}} B_{1} \\
0 & A_{11} & A_{12} & \frac{1}{\sqrt{2}} B_{1} \\
0 & A_{21} & A_{22} & \frac{1}{\sqrt{2}} B_{2} \\
-\frac{1}{\sqrt{2}} C_{1} & \frac{1}{\sqrt{2}} C_{1} & \frac{1}{\sqrt{2}} C_{2} & 0
\end{array}\right] .
$$

Motivated by the results of Lemmas 23 and 24, we consider the following similarity transformation, shown in:

$$
T=\left[\begin{array}{cccc}
-\frac{1}{\sqrt{2}} \Sigma_{1}^{1 / 2} & \frac{1}{\sqrt{2}} \Sigma_{1}^{1 / 2} & 0 & 0 \\
0 & 0 & I & 0 \\
\frac{1}{\sqrt{2}} \Sigma_{1}^{-1 / 2} & \frac{1}{\sqrt{2}} \Sigma_{1}^{-1 / 2} & 0 & 0 \\
0 & 0 & 0 & I
\end{array}\right]
$$

giving (29) shown at the bottom of the page. To prove the main result, we will show that $T M T^{-1}$ is contractive, and hence $M$ is contractive. Note that

$$
T M T^{-1}=\left[\begin{array}{llll}
0 & 0 & I & 0 \\
0 & I & 0 & 0 \\
I & 0 & 0 & 0 \\
0 & 0 & 0 & I
\end{array}\right] \hat{M}
$$

where $\hat{M}$ is shown at the bottom of the page in (30). Let

$$
\begin{aligned}
U_{11} & =\Sigma_{1}^{-1 / 2} A_{12} \\
U_{12} & =\left[\begin{array}{ll}
\Sigma_{1}^{-1 / 2} A_{11} \Sigma_{1}^{1 / 2} & \Sigma_{1}^{-1 / 2} B_{1}
\end{array}\right] \\
U_{22} & =\left[\begin{array}{ll}
A_{21} \Sigma_{1}^{1 / 2} & B_{2}
\end{array}\right] \\
V_{11} & =A_{21} \Sigma_{1}^{-1 / 2} \\
V_{21} & =\left[\begin{array}{c}
\Sigma_{1}^{1 / 2} A_{11} \Sigma_{1}^{-1 / 2} \\
C_{1} \Sigma_{1}^{-1 / 2}
\end{array}\right] \\
V_{22} & =\left[\begin{array}{c}
\Sigma_{1}^{1 / 2} A_{12} \\
C_{2}
\end{array}\right] \\
Z & =A_{22} .
\end{aligned}
$$

and

Note that

and

$$
\begin{aligned}
& U=\left[\begin{array}{cc}
U_{11} & U_{12} \\
Z & U_{22}
\end{array}\right] \\
& V=\left[\begin{array}{cc}
V_{11} & Z \\
V_{21} & V_{22}
\end{array}\right]
\end{aligned}
$$

are contractive by Lemma 24. Applying Lemma 23 shows that $\hat{M}$ is contractive, and therefore $M$ is contractive. Thus $\frac{1}{2}\left\|G-G_{r}\right\|_{\infty} \leq 1$ by Lemma 22 .

The proof for Theorem 15, for which we provide an outline, follows from a strict generalization of the proof given for the 1-D case.

Proof: By repeated permutations, scalings and truncations, we can apply the methods of Theorem 5 along with the sufficiency direction of Lemma 14 to obtain the stated bound.

$$
\begin{aligned}
T M T^{-1} & =\left[\begin{array}{ccccc}
\Sigma_{1}^{1 / 2} A_{11} \Sigma_{1}^{-1 / 2} & \frac{1}{\sqrt{2}} \Sigma_{1}^{1 / 2} A_{12} & 0 & 0 \\
\frac{1}{\sqrt{2}} A_{21} \Sigma_{1}^{-1 / 2} & A_{22} & \frac{1}{\sqrt{2}} A_{21} \Sigma_{1}^{1 / 2} & \frac{1}{\sqrt{2}} B_{2} \\
0 & \frac{1}{\sqrt{2}} \Sigma_{1}^{-1 / 2} A_{12} & \Sigma_{1}^{-1 / 2} A_{11} \Sigma_{1}^{1 / 2} & \Sigma_{1}^{-1 / 2} B_{1} \\
C_{1} \Sigma_{1}^{-1 / 2} & \frac{1}{\sqrt{2}} C_{2} & 0 & 0
\end{array}\right] \\
\hat{M} & =\left[\begin{array}{cccccc}
0 & \vdots & \frac{1}{\sqrt{2}} \Sigma_{1}^{-1 / 2} A_{12} & \vdots & \Sigma_{1}^{-1 / 2} A_{11} \Sigma_{1}^{1 / 2} & \Sigma_{1}^{-1 / 2} B_{1} \\
\ldots & \cdots & \cdots & \cdots & \cdots & \cdots \\
\frac{1}{\sqrt{2}} A_{21} \Sigma_{1}^{-1 / 2} & \vdots & A_{22} & \vdots & \frac{1}{\sqrt{2}} A_{21} \Sigma_{1}^{1 / 2} & \frac{1}{\sqrt{2}} B_{2} \\
\ldots & \cdots & \cdots & \cdots & \cdots & \cdots \\
\Sigma_{1}^{1 / 2} A_{11} \Sigma_{1}^{-1 / 2} & \vdots & \frac{1}{\sqrt{2}} \Sigma_{1}^{1 / 2} A_{12} & \vdots & 0 & 0 \\
C_{1} \Sigma_{1}^{-1 / 2} & \vdots & \frac{1}{\sqrt{2}} C_{2} & \vdots & 0 & 0
\end{array}\right]
\end{aligned}
$$




$$
M_{I}=\left[\begin{array}{cccccc}
0 & \vdots & \frac{1}{\sqrt{2}} \hat{\Sigma}^{-1 / 2} A_{12} & \vdots & \hat{\Sigma}^{-1 / 2} \hat{A} \hat{\Sigma}^{1 / 2} & \hat{\Sigma}^{-1 / 2} \hat{B} \\
\ldots & \ldots & \ldots & \ldots & \ldots & \ldots \\
\frac{1}{\sqrt{2}} A_{21} \hat{\Sigma}^{-1 / 2} & \vdots & A_{22} & \vdots & \frac{1}{\sqrt{2}} A_{21} \hat{\Sigma}^{1 / 2} & \frac{1}{\sqrt{2}} B_{2} \\
\ldots & \ldots & \ldots & \ldots & \ldots & \ldots \\
\hat{\Sigma}^{1 / 2} \hat{A} \hat{\Sigma}^{-1 / 2} & \vdots & \frac{1}{\sqrt{2}} \hat{\Sigma}^{1 / 2} A_{12} & \vdots & 0 & 0 \\
\hat{C} \hat{\Sigma}^{-1 / 2} & \vdots & \frac{1}{\sqrt{2}} C_{2} & \vdots & 0 & 0
\end{array}\right]
$$

That is, we assume the system realization is reduced from

$$
M=\left[\begin{array}{ccc}
\hat{A} & A_{12} & \hat{B} \\
A_{21} & A_{22} & B_{2} \\
\hat{C} & C_{2} & D
\end{array}\right]
$$

to $M_{r}=\left[\begin{array}{ll}\hat{A} & \hat{B} \\ \bar{C} & D\end{array}\right]$, with $\Sigma=\operatorname{diag}[\hat{\Sigma}, I]$. The corresponding uncertainty structure is reduced from $\Delta=\operatorname{diag}\left[\delta_{1} I_{n_{1}}, \cdots, \delta_{p} I_{n_{p}}\right]$ to $\Delta_{r}=\operatorname{diag}\left[\delta_{1} I_{n_{1}}\right.$, $\left.\cdots, \delta_{p-1} I_{n_{p-1}}, \delta_{p} I_{\hat{n}_{p}}\right]$, where $\hat{n}_{p}=\sum_{j=1}^{\left(t_{p}-1\right)} s_{p j}<n_{p}$, that is, only the representation submatrices corresponding to the last uncertainty variable $\delta_{p}$ in $\Delta$ and the last singular value $\sigma_{p t_{p}}$ in $\Sigma_{p}$ are reduced. As in the 1-D case, we assume $\sigma_{p t_{p}}=1$ and subsequently show that $\frac{1}{2}\left\|(\Delta \star M)-\left(\boldsymbol{\Delta}_{r} \star M_{r}\right)\right\|_{\mathrm{SI} 2} \leq 1$.

Let

$$
\tilde{M}=\left[\begin{array}{cccc}
\hat{A} & 0 & 0 & \frac{1}{\sqrt{2}} \hat{B} \\
0 & \hat{A} & A_{12} & \frac{1}{\sqrt{2}} \hat{B} \\
0 & A_{21} & A_{22} & \frac{1}{\sqrt{2}} B_{2} \\
-\frac{1}{\sqrt{2}} \hat{C} & \frac{1}{\sqrt{2}} \hat{C} & \frac{1}{\sqrt{2}} C_{2} & 0
\end{array}\right]
$$

and $\tilde{\Delta}=\operatorname{diag}\left[\Delta_{r}, \Delta\right]$, with corresponding commutative matrix set $\tilde{\mathcal{T}}$. Using a similarity transformation $T$ like that in the proof of Theorem 5 gives

$$
T \tilde{M} T^{-1}=\left[\begin{array}{llll}
0 & 0 & I & 0 \\
0 & I & 0 & 0 \\
I & 0 & 0 & 0 \\
0 & 0 & 0 & I
\end{array}\right] M_{I}
$$

where $M_{I}$ is shown at the top of the page in (31). Now defining $U$ and $V$ as in Theorem 5 and using Lemma 24 and the fact that the Lyapunov inequalities are satisfied by assumption gives us that $U$ and $V$ are contractive. Applying Lemma 23 then implies that $M_{I}$ is contractive, and hence $\tilde{M}$ is contractive. Finally, from Lemma 14 we have that $\frac{1}{2}\|(\tilde{\boldsymbol{\Delta}} \star \tilde{M})\|_{\text {SI2 }} \leq 1$.

Remark 25: Note that although the similarity transformation $T$ used in the preceding proof is not strictly an element of $\tilde{\mathcal{T}}$, it can be written as the composition of an element of $\tilde{\mathcal{T}}$ and a permutation and thus is allowable.

\section{ACKNOWLEDGMENT}

The authors are grateful to the reviewers for their helpful comments and suggestions.

\section{REFERENCES}

(1) L. Andersson and C. Beck, "Model comparison and simplification," accepted for 35th Conf. Decision Contr., Kobe, Japan, 1996.

[2] P. Apkarian and P. Gahinet, "A convex characterization of gainscheduled H-infinity controllers," IEEE Trans. Automat. Contr., vol. 40, pp. 853-864, 1995 .

131 C. Beck, "Model reduction and minimality for uncertain systems," Ph.D. dissertation, California Instit. Technol., 1996.

4] P. Bendotti and C. Beck, "On the role of LFT model reduction methods in robust controller synthesis for a pressurized water reactor," 1996. report in preparation.

[5] C. Beck and J. C. Doyle. "A necessary and sufficient minimality condition for uncertain systems," IEEE Trans. Automat. Contr., submitted.

[6] ___ "Realizations of uncertain systems and formal power series," in Proc. 1995 Amer. Contr. Conf., WA, 1995.

[7] _ _ "Model reduction of behavioral systems," in Proc. 32nd Conf. Decision Contr., TX, 1993.

[8] J. Berstel and C. Reutenauer, "Rational series and their languages," Monographs on Theoretical Computer Science. New York: SpringerVerlag, 1988.

[9] N. K. Bose, Applied Muhidimensional System Theory. New York: Van Nostrand Reinhold, 1982.

[10] S. Boyd and Q. Yang. "Simultaneous quadratic Lyapunov functions," Stanford Research Tech. Rep., 1988.

(11] J. David and B. DeMoor, "The opposite of analytic centering for solving minimum rank problems in control and identification," in Proc. 32nd Conf. Decision Contr. TX, 1993.

[12] — , "Designing reduced order output feedback controllers using a potential reduction method," in Proc. 1994 Amer. Contr. Conf., MD, 1994.

[13] U. B. Desai and D. Pal, "A transformation approach to stochastic model reduction," IEEE Trans. Automat. Contr, vol. AC-29, pp. 1097-1100, 1984.

[14] J. Doyle, A. Packard, and K. Zhou, "LFT's, LMI's, and $\mu$," in Proc. 30th Conf. Decision Contr., Brighton, UK, 1991.

[15] D. F. Enns, "Model reduction for control system design," Ph.D. dissertation, Stanford Univ., 1984

[16] L. El Ghaoui, F. Delebecque, and R. Nikoukah, LMITOOL: A UserFriendly Interface for LMI Optimization, 1995.

[17] P. Gahinet, A. Nemirovskii, A. J. Laub, and M. Chilali, The LMI Control Toolbox. S. Natick, MA: The MathWorks, 1994.

[18] K. Glover, "All optimal Hankel-Norm approximations of linear multivariable systems and their $L^{\infty}$-error bounds," Int. J. Contr., vol. 39 , pp. [115-1193, 1984.

[19] M. Fliess, "Matrices de Hankel," J. Math. pures et appl., vol. 53, pp. $97-222,1974$.

[20] A. Helmersson, "Methods for robust gain scheduling," Ph.D. dissertation, Linköping Univ., 1995.

[21] D. Himrichsen and A. J. Pritchard, "An improved error estimate for reduced-order models of discrete-time systems." IEEE Trans. Automat. Contr. vol. 35, pp. 317-320, 1990. 
[22] T. Iwasaki and R. E. Skelton, "All controllers for the general $\mathcal{H}_{\infty}$ problem: LMI existence conditions and state space formulas," Syst. Contr. Lett., vol. 30, pp. 1307-1317, 1994.

[23] D. Kavranoglu and M. Bettayeb, "Characterization of the solution to the optimal $H_{\infty}$ model reduction problem," Syst. Contr. Lett., vol. 20, pp. 99-107, 1993

[24] A. Megretskii, "Necessary and sufficient conditions of stability: A multiloop generalization of the circle criterion," IEEE Trans. Automat. Contr., vol. 38, pp. 753-756, 1993.

[25] D. G. Meyer, "A fractional approach to model reduction," in Proc. Amer. Contr. Conf., Atlanta, GA, 1988.

[26] B. C. Moore, "Principal component analysis in linear systems: Controllability, observability and model reduction," IEEE Trans. Automat. Contr., vol. AC-26, pp. 17-32, 1981.

[27] A. Packard, K. Zhou, P. Pandey, J. Leonhardson, and G. Balas, "Optimal, constant $\mathrm{I} / \mathrm{O}$ similarity scaling for full-information and statefeedback control problems," Syst. Contr. Lett., vol. 19, pp. 271-280, 1992.

[28] A. Packard, "Gain scheduling via linear fraction transformations," Syst. Cont. Lett., vol. 22, pp. 79-92, 1994.

[29] F. Paganini, "Sets and constraints in the analysis of uncertain systems," Ph.D. dissertation, California Inst. Technol., 1995.

[30] A. Rantzer, "Error bounds for nonlinear model reduction," 1995, preprint.

[31] J. Shamma, "Robust stability with time-varying structured uncertainty," IEEE Trans., Automat. Contr. vol. 39, pp. 714-724, 1994.

[32] W. Wang, J. Doyle, C. Beck, and K. Glover, "Model reduction of LFT systems," in Proc. 30th Conf. Decision Contr., Brighton, England, 1991.

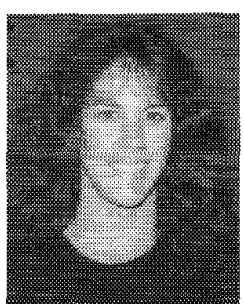

Carolyn L. Beck received the B.S. degree from California State Polytechnic University, Pomona, in 1984 and the M.S degree from Carnegie Mellon University in 1985, both in electrical and computer engineering. She received the Ph.D. degree in electrical engineering from the California Institute of Technology in 1996.

From 1985-1989 she was employed as a Research and Development Engineer for Hewlett-Packard in Santa Clara, CA. In 1996 she was a Postdoctoral Research Assistant at the Lund Institute of 'Technology in Sweden. She has recently joined the faculty of the Department of Electrical Engineering at the University of Pittsburgh. Her research interests include modeling and control of uncertain and nonlinear systems.

John Doyle, for a photograph and biography, see p. 65 of the January 1996 issue of this TRANSACTIONS.

Keith Glover (S'71-M'73-SM'90-F'93), for a photograph and biography, see p. 1159 of the August 1996 issue of this Transactions. 